\title{
On the long-term evolution of the sensitivity of the STEREO HI-1 cameras
}

\author{
S.J. Tappin, C.J. Eyles and J.A. Davies
}

\section{Published version information}

Citation: SJ Tappin, CJ Eyles, and JA Davies. "On the long-term evolution of the sensitivity of the STEREO HI-1 cameras." Solar Physics, vol. 292, no. 2 (2017): 28. doi: $\underline{10.1007 / \mathrm{s} 11207-017-1052-0}$

The final publication is available at Springer via http://dx.doi.org/10.1007/s11207$\underline{017-1052-0}$

This version is made available in accordance with publisher policies. Please cite only the published version using the reference above. 


\title{
On the Long-Term Evolution of the Sensitivity of the STEREO HI-1 Cameras.
}

\author{
S.J. Tappin ${ }^{1} \cdot$ C.J. Eyles ${ }^{1,2}$ - J.A. Davies ${ }^{1}$ \\ (C) Springer
}

\begin{abstract}
The Heliospheric Imagers (HI) on the Solar TErrestrial RElations Observatory (STEREO) observe the solar wind and disturbances therein as it propagates from close to the Sun to $1 \mathrm{AU}$ and beyond. In this article we use stellar photometry over much of the mission to date to make a determination of the long-term evolution of the photometric response of the inner (HI-1) cameras. We find very slow degradation rates of the order of $0.1 \%$ per year, similar to those found for HI-2 by Tappin, Eyles and Davies (2015, Solar Phys. 290, 2143) and significantly slower than other comparable instruments. We also find that it is necessary to make a small $(\approx 1 \%)$ revision to the photometric calibration parameters used to convert instrument units to physical units. Finally, we briefly discuss the effects of pointing instabilities on the measurement of stellar count rates.
\end{abstract}

Keywords: Instrumental Effects; Instrumentation and Data Management

\section{Introduction}

The Solar Terrestrial Relations Observatory (STEREO: Kaiser et al., 2008), launched in late 2006, is a two-spacecraft NASA mission to investigate (inter alia) the initiation and propagation of solar coronal mass ejections (CMEs) from locations separated in ecliptic longitude. The two spacecraft were placed

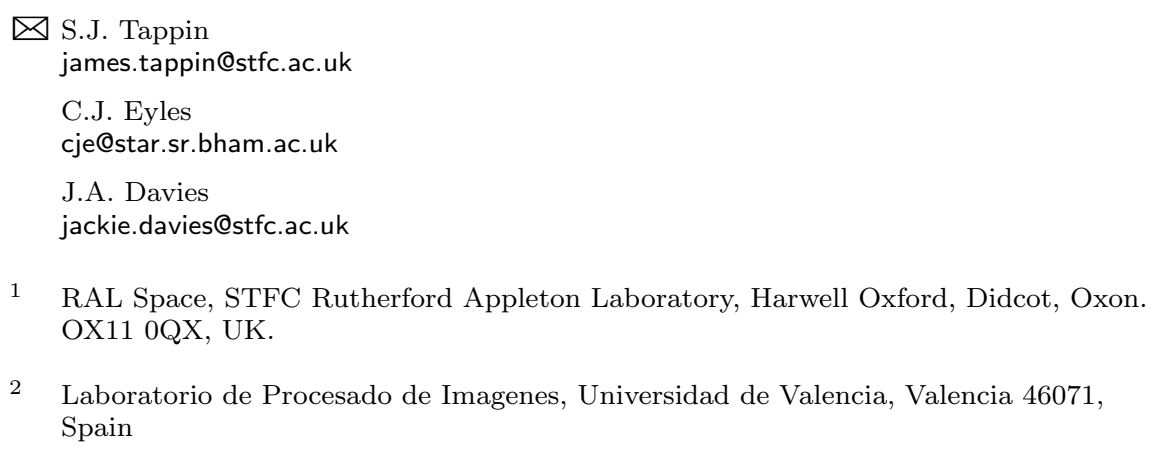


in heliocentric orbits, one (the ahead spacecraft; STEREO-A) somewhat inside $1 \mathrm{AU}$ and the other (the behind spacecraft; STEREO-B) somewhat outside, such that the spacecraft drift ahead of and behind the Earth by about $22^{\circ}$ per year. Full science operations began in April 2007. The STEREO spacecraft reached solar superior conjunction in early 2015. For an interval of approximately one year around conjunction, the small angular separation of the spacecraft and the Sun meant that limitations on telemetry forced a reduced observation program. STEREO-A has now emerged from conjunction and resumed full operation. Unfortunately, contact with STEREO-B was lost shortly before conjunction. Signals from STEREO-B were detected during August and September of 2016, but attempts to regain control of the spacecraft attitude were not successful. Further attempts are planned for 2017, when it is expected that the orientation of the spacecraft rotation axis relative to the Earth and the Sun will provide a more favourable power balance and communication alignment.

The imaging capabilities of the STEREO spacecraft are provided by the Sun Earth Connection Coronal and Heliospheric Investigation (SECCHI: Howard et al., 2008), which is a package of instruments consisting of an extreme ultraviolet imager, two coronagraphs, and two Heliospheric Imagers. The Heliospheric Imagers (HI: Eyles et al., 2009) use Thomson-scattered sunlight to detect and track CMEs and other solar-wind disturbances from the outer limits of the coronagraph fields of view out to $1 \mathrm{AU}$ and beyond. The HI cameras have nominally circular fields of view offset from the Sun to the earthward side. The inner (HI-1) cameras have fields of view that are $20^{\circ}$ in diameter, centred at an elongation of $14^{\circ}$. The outer (HI-2) instruments have $70^{\circ}$ diameter fields centred at an elongation of $53^{\circ}$. In normal operations, HI-1 takes one image every 40 minutes (36 per day) and HI-2 takes one every two hours.

It is generally expected that the detectors on space-based imaging instruments will degrade slowly over the course of their lifetime (BenMoussa et al., 2013). In earlier studies of the evolution of HI-1 sensitivity (Bewsher, Brown, and Eyles, 2012; BenMoussa et al., 2013) no measurable degradation was found and an upper limit of about $1 \%$ over an interval of about four years was placed on the degradation rate. However, for the HI-2 cameras Tappin, Eyles, and Davies (2015) found degradation rates of about $0.1 \%$ per year. In this article we use an extension of the techniques used by those authors for HI-2 to obtain values for the degradation rates for HI-1. We also compute a minor revision to the photometric calibration parameters compared with those of Bewsher et al. (2010) and Bewsher, Brown, and Eyles (2012). This was driven by both an improved understanding of the HI-1 data and also an increased dataset. In Appendix A we provide a brief discussion of the effects of pointing shifts on the measured counting rates of stars, and the implications for this calibration.

\section{Notes on terminology:}

- Since the science images from the HI cameras are $2 \times 2$ binned before transmission to Earth (Eyles et al., 2009), it is important to maintain the distinction between pixels on the $2048 \times 2048$ camera CCD and pixels in the $1024 \times 1024$ science images. Therefore, in this article (as in Tappin, Eyles, and Davies, 2015,) we will refer to a pixel on the CCD as a pixel, 
and one in a science image as a bin. In general, our analyses are presented in terms of CCD pixels with conversions to image bins provided when this is applicable.

- To make a distinction with the actual gain of the instruments (when observing diffuse structures) we use the term "apparent gain" to refer to the gain inferred from stellar photometry without correction for the various errors described in this article.

\section{Methods}

The methods used, which are described in this section, can be thought of as a synthesis of those used for the original HI-1 photometric calibrations by Bewsher et al. (2010) and those used for HI-2 by Tappin, Eyles, and Davies (2015). In particular, Tappin, Eyles, and Davies (2015) developed a method to reduce the variation between stars that allowed the detection of very small changes in sensitivity, thus opening up the possibility of improving on the limits found by Bewsher, Brown, and Eyles (2012) and BenMoussa et al. (2013).

\subsection{Stellar Sample}

Since the $10^{\circ}$ radius field of view of the HI- 1 cameras is much smaller than that of HI-2, the Yale bright star catalogue (Hoffleit and Warren, 1995) used by Tappin, Eyles, and Davies (2015) does not contain enough stars that pass through the HI-1 field of view for a satisfactory photometric calibration. Equally, we find that the NOMAD catalogue (Zacharias et al., 2004) used by Bewsher et al. (2010) and Bewsher, Brown, and Eyles (2012) is not particularly convenient for our purposes, as spectral information must be obtained from other sources. We have therefore used the SKY2000 catalogue (Myers et al., 2001), which includes stars down to about tenth magnitude, and which includes spectral information as well as other required parameters. From this, we defined an initial sample by applying a number of selection criteria. These are similar to those of Tappin, Eyles, and Davies (2015) apart from the magnitude range and minimum angular separation between stars, which are fainter and smaller respectively, to account for the smaller field of view and point spread function of HI-1.

- The star must not be a double (whether binary or optical).

- It must not be listed as variable.

- It must pass within 200 image bins of the centre of the HI-1 field of view.

- It must have a HI-1A photonic magnitude between 4.0 and 8.5. Photonic magnitude takes account of the fact that the CCD detectors used in HI respond to the number of detected photons rather than bolometric intensity. It also factors in the difference between the HI-1 passband and the standard astronomical $\mathrm{V}$ passband. The details of the derivation of photonic magnitude are given by Tappin, Eyles, and Davies (2015).

- It must not lie within $0.2^{\circ}$ of another star in the catalogue (i.e. a star brighter than tenth magnitude). 
- It must not have a peculiar or variable spectral type.

- It must have a spectral type that can be matched to a spectrum in Pickles' collection of stellar spectra (Pickles, 1998). A spectral match is considered valid if: the luminosity class is a single value (e.g. stars with luminosity class III-IV would be rejected as Pickles (1998) does not have spectra for such cases), and one of the following is satisfied:

i) There is an exact match to a type with a spectrum.

ii) The spectral type lies within a range that shares a common spectrum in the Pickles (1998) catalogue (e.g. Pickles (1998) lists a single spectrum for B1-2 III stars that would be used for both B1 III and B2 III).

iii) The spectral type is a range that spans a spectrum in Pickles (1998) (e.g. a star listed as G8-K0 III could match any of G8 III, G9 III or K0 III)

iv) The spectral type can be matched by interpolating between two spectra separated by no more than three subclasses (e.g. K4 could be derived from $\mathrm{K} 2$ and $\mathrm{K} 5$, but not from $\mathrm{K} 2$ and $\mathrm{K} 6$ ).

In all cases, an exact luminosity class match is required (e.g. we would not attempt to interpolate between A2 I and A2 III to obtain a spectrum for A2 II; we do however consider the supergiant classes I, Ia and Ib to be equivalent). Unlike the analysis of Bewsher et al. (2010) and Bewsher, Brown, and Eyles (2012), it was not necessary to resort to colour mixing as spectral types were available for all of the stars in our sample and the vast majority could be matched to spectra in Pickles (1998); those few that could not be adequately matched to a spectrum in Pickles (1998) were discarded.

This set of criteria produced an initial sample of 1504 stars that could potentially be used for calibration of HI-1A and 1570 for HI-1B.

\subsection{Photometry}

\subsubsection{Introduction}

The dataset used for the analyses presented in this article was the Level-2 science images in units of $\mathrm{DN} \mathrm{s}^{-1}$ (CCD pixel) $)^{-1}$ with a one-day background subtracted, as was used for the earlier HI-1 photometric calibrations by Bewsher et al. (2010) and Bewsher, Brown, and Eyles (2012). These images can be obtained from the UK Solar System Data Centre (UKSSDC: www.ukssdc.ac.uk/solar/ stereo/data.html). The processing is described on the UKSSDC web site (www. ukssdc.ac.uk/solar/stereo/documentation/HI_processing.html and www.ukssdc.ac. uk/solar/stereo/documentation/HI_processing_L2_data.html). In summary: the raw images are converted from units of data numbers (DN) to units of DN s ${ }^{-1}(\mathrm{CCD} \text { pixel })^{-1}$, the readout smearing caused by the shutterless operation (Eyles et al., 2009) is removed, columns containing severely saturated bins are set to NaN values, the large-scale flatfield correction determined by Bewsher et al. (2010) is applied, and the pointing information in the header is corrected by aligning with the starfield (Brown, Bewsher, and Eyles, 2009) to generate the Level-1 images. Finally a oneday running mean of the lowest quartile of the measurements in each individual 
Table 1. Summary of the apertures and background annuli used to determine the aperture photometry curves of growth shown in Figure 1; all values are in image bins.

\begin{tabular}{rrrrrr} 
Group & Min & Max & Radius & \multicolumn{2}{c}{ Background } \\
& Radius & Radius & Step & $\begin{array}{r}\text { Inner } \\
\text { Outer }\end{array}$ & $\begin{array}{r}\text { Oudius } \\
\text { Radius }\end{array}$ \\
\hline 1 & 1.0 & 3.0 & 0.2 & 4.0 & 8.0 \\
2 & 3.0 & 5.0 & 0.2 & 6.0 & 10.0 \\
3 & 5.0 & 7.0 & 0.2 & 8.0 & 12.0 \\
4 & 7.0 & 12.0 & 0.5 & 13.0 & 16.0 \\
\hline
\end{tabular}

bin is subtracted to produce the Level-2 images. It should be noted that at the time of the analyses of Bewsher et al. (2010) and Bewsher, Brown, and Eyles (2012) a one-day running minimum was used as a background; however, since the photometry method used for those studies and also in this article includes a local background subtraction, we think it unlikely that this change will have made a significant difference to the photometric calibration parameters.

After some experimentation, we found that the standard aperture photometry methods (Stetson, 1987) used by Bewsher et al. (2010) gave more consistent count-rate measurements than the gradient-based method developed by Tappin, Eyles, and Davies (2015) for HI-2. This is to be expected, as that method was devised to deal with the variable point spread function (PSF) of the HI-2 instruments, and the HI-1 PSF is much less variable across the field of view.

\subsubsection{Aperture Selection}

To find the optimal photometry aperture, we generated curves of growth of the measured count rates for both HI-1A and HI-1B as a function of the photometry aperture radius. For this analysis, we used the same sample of stars that is described in Section 2.1, and also made a number of magnitude-limited subsamples. Since computing the curve from the entire dataset would have been impractical, we used data from 2009 and 2010 in order to avoid some of the issues with HI-1B pointing early in the mission (Appendix A.2). We used a range of aperture radii from 1.0 to 12.0 bins, with appropriate background annuli as summarized in Table 1. Note that in order to assess the effect of the background annulus on the photometry, we repeated the largest aperture radius of a group at the start of the next group.

For each star, the median count rate over the two-year interval was determined for each aperture. Then, to allow comparison between stars, all of the rates for each star were normalized to the rate from the 1.6 bin aperture. Finally, to produce a curve of growth as a function of photometry aperture we took the median of these normalized rates at each aperture. The results of this, for apertures up to a radius of 8 bins, are shown as the solid lines in Figure 1. At small apertures, the normalized count rate increases rapidly with aperture size as expected, as more of the PSF is encompassed by the photometry aperture. 
a)

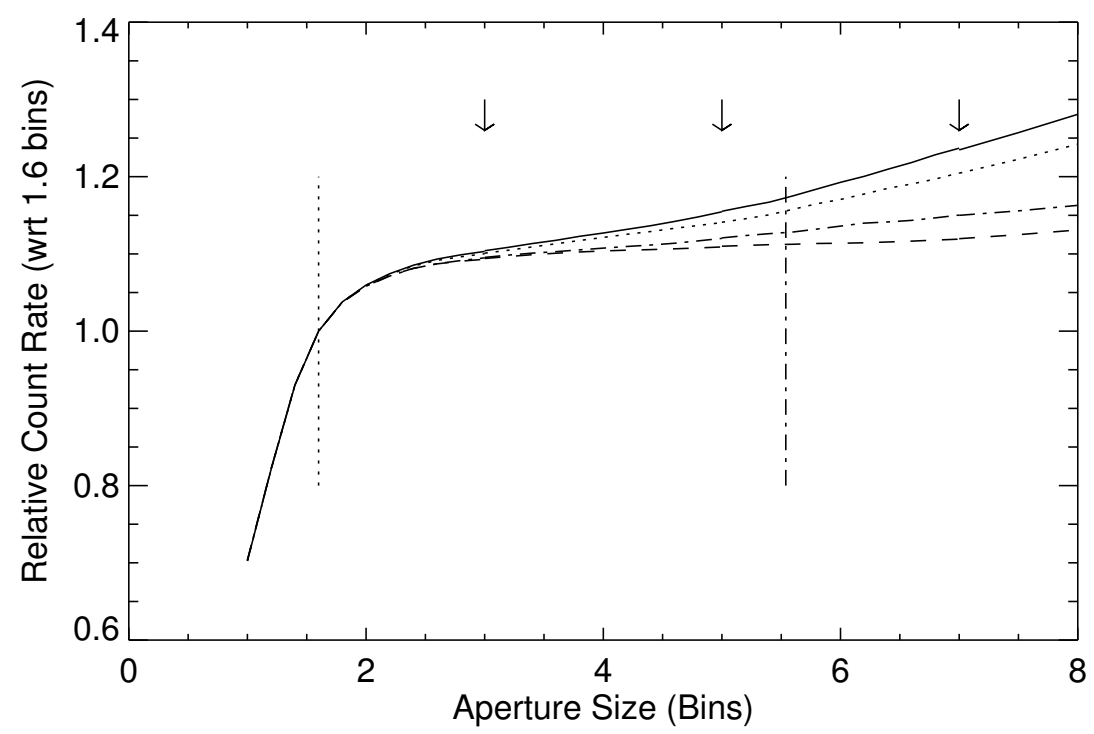

b)

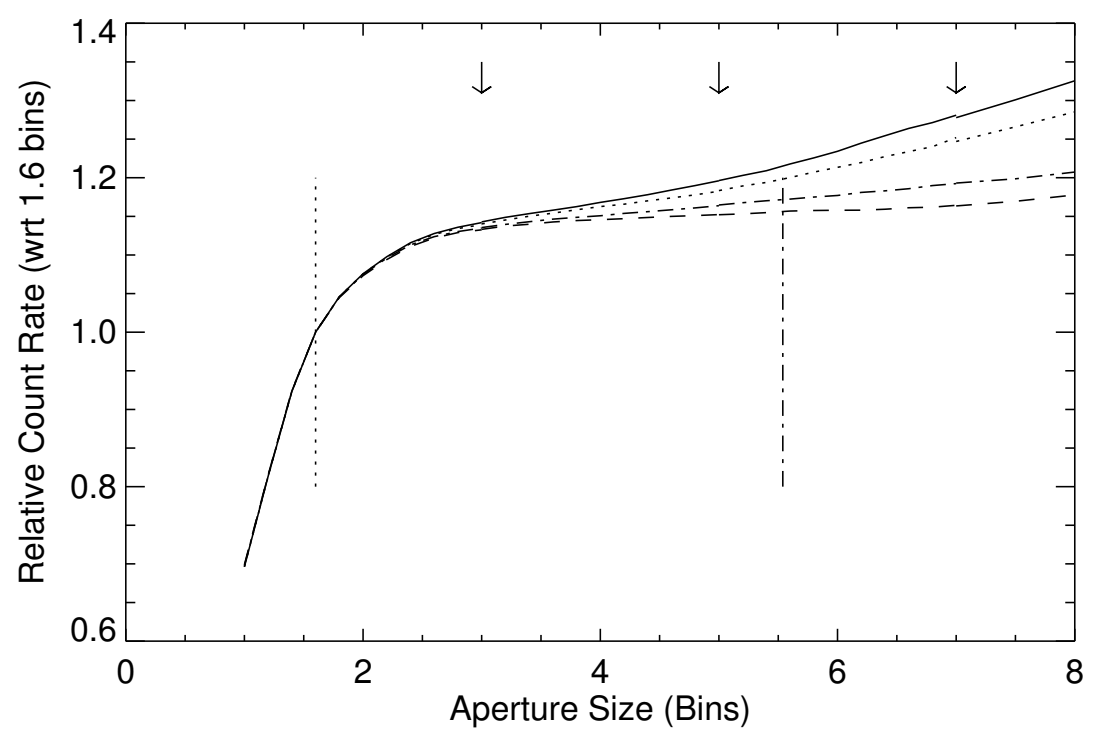

Figure 1. Aperture photometry curves of growth for: a) HI-1A and b) HI-1B. Solid lines = All Stars; Dashed lines = Stars brighter than sixth magnitude; Dash-dot lines = Stars brighter than seventh; and Dotted lines = Stars brighter than eighth magnitude (the curve for stars brighter than fifth magnitude is omitted as it is very similar to that for stars brighter than sixth magnitude over the range of apertures shown here). The vertical dotted line indicates the 1.6 bin aperture used for normalization, the vertical dash-dot line indicates the $0.2^{\circ}$ minimum separation limit from other catalogue stars, and the arrows at the top indicate the apertures at which the background annulus was changed. 
This increase then slows at about 2.5 bins, but it starts to accelerate again beyond about 4 bins (between 4 and 5 arcmin). We note that the discontinuities where the background annulus was changed are very small $(\ll 1 \%)$. This implies that the choice of background annulus is not critical, which we might expect as the background removal in the Level-2 processing should remove any large-scale gradients.

To understand the increase in relative count rate at large apertures, it is necessary to consider how the aperture-photometry method works. The stellar count rate is computed by summing the counts in all of the bins within the aperture with a weighting factor for those bins that are only partially within the aperture. Thus any additional stars or confusion fluctuations (Scheuer, 1957) are added into the stellar count rate. The background rate, on the other hand, is computed as an estimate of the mode of the distribution of count rates in the background annulus (using the SolarSoft DAOPHOT routine $\mathrm{mmm}$ ) multiplied by the number of pixels in the photometry aperture. This allows a reliable estimate of the background even where there are significant stars in the background annulus, as the mode of a distribution is less sensitive to outliers that either the mean or the median (Stetson, 1987). This does however mean that if there is a significant contribution from faint stars in the photometry aperture, this is not removed by the background. We thus interpret the increase in relative count rate at large apertures as the result of faint stars, below the limit of the SKY2000 catalogue but above the confusion limit, which are added into the count rate but effectively excluded from the background rate. To verify this, we re-ran the curve of growth computations restricting the analyses to stars brighter than fifth, sixth, seventh and eighth magnitude. The last three of these are shown as the various broken curves in Figure 1. The fact that, for brighter stars, the increase at large apertures is much slower than for fainter stars supports our explanation of its cause.

These results would suggest that, ideally, we should restrict our analysis to only the brightest stars in the sample. However, there are only 59 stars of magnitude 6.0 and brighter in our calibration sample. Moreover, we have also found that the brighter stars have systematically reduced count rates relative to their predicted rates as a result of cosmic ray scrubbing (see: Appendix A, and Tappin (2017), and to a lesser extent saturation.

From the curves in Figure 1, we consider that the cross-over between missing flux, because the aperture is smaller than the PSF, and gaining spurious flux from faint stars in the photometry aperture is close to three bins and that at this radius both contributions appear to be small. We therefore use an aperture of 3.0 bins, with a background annulus from 5.0 to 10.0 bins, for the remaining analyses presented in this article. This aperture is slightly smaller than the 3.2 (HI-1A) and 3.1 (HI-1B) bin apertures used by Bewsher et al. (2010), Bewsher, Brown, and Eyles (2012), and BenMoussa et al. (2013); however we consider that the increase of confusion flux with aperture makes it desirable to use as small an aperture as possible without excluding counts from the edge of the PSF. 


\subsubsection{Measurements and Post-Measurement Selections}

Measurements of all of the stars in our sample were made for every image in which those stars were within 200 image bins of the image centre, from the start of regular science operations in April 2007 until the start of reduced operations prior to superior conjunction for STEREO-A on 19 August 2014, and until the loss of contact with STEREO-B on 1 October 2014.

We then restricted our analysis to stars satisfying the following additional criteria:

i) The median integrated count rate for the star must not exceed $400 \mathrm{DN} \mathrm{s}^{-1}$. This limi,t which corresponds to about magnitude 5.5, is imposed as there is a significant reduction of the observed count rates relative to the predicted values above this level. We believe that this is caused by the movement of the stars through the field of view generating false positives in the cosmic-ray scrubbing algorithm (Appendix A.3).

ii) There must be at least 100 measurements of the star within the 200-bin radius core region.

iii) The star must be measured in at least three orbits, covering an interval of 3.5 years or more. This ensures that the time span for the star is at least four orbits (e.g., a star appearing in Orbits 2, 3, and 4 would not be used but one appearing in 2, 4, and 5 would be used). In practice the majority of the stars eliminated by this criterion (and the previous one) are those stars that only pass within 200 bins of the image centre in the first STEREO orbit when spacecraft roll values (especially for STEREO-B) were large.

iv) The interquartile range of the count rate measurements of the star must not exceed 0.02 of the median count rate, i.e. there is little scatter in the count rate measurements.

This left 1422 out of the original 1504 stars suitable for this analysis in HI-1A, and 1417 of the original 1570 for HI-1B. The differences in numbers stem from the larger roll of STEREO-B early in the mission, which meant that a significant number of stars passed through the inner 200 bins of HI-1B on the first orbit only.

In addition, during the processing and analysis, it became clear that there were intervals of anomalously low apparent gain:

i) Late in the observation series there was a drop in the apparent gain of both HI-1A and HI-1B. These were found to match the times at which the attitude control gyros were turned off and the attitude control loop was closed solely around the guide telescope and star trackers, namely 18 September 2013 for STEREO-A and 7 January 2014 for STEREO-B. This resulted in significantly degraded stability about the spacecraft roll axis (although still within the original specification).

ii) In HI-1B there were a number of dips in the apparent gain, each lasting a few days. These mainly occurred early in the mission, and were mostly around the times of spacecraft momentum dumps. 
We discuss these gain anomalies in more detail in Appendix A, but in both cases pointing shifts on the time-scale of the individual exposures within a summed exposure sequence, from which a science image is assembled (Eyles et al., 2009), cause the onboard cosmic-ray scrubbing algorithm to misidentify pixels containing stellar flux as particle hits. For the purposes of this study, we therefore excluded measurements after the switch to gyroless attitude control. We also eliminated other intervals where the spread of the particle hit counts was high $(\Delta>0.15$, see Equation (4) below in Appendix A.2), which we found to be a good proxy for reduced apparent gain.

\section{Results}

\subsection{Gain Correction Factors}

Since we are using a different sample of stars and different statistical techniques from Bewsher et al. (2010) and Bewsher, Brown, and Eyles (2012), we need to compare our results for the overall adjustments to the pre-launch gains prior to fitting the degradation rates. A revision is also appropriate as we now have more data available to us than were available to those authors. Furthermore, they were not aware of the effects of the HI-1B pointing shifts on measured stellar count rates, nor of the effect of the orbital motion of the spacecraft on the cosmic-ray scrubbing (see Appendix A.2 and A.3, respectively). Their measurements were, however, entirely prior to the switch to gyroless attitude control. In this article, we have only computed on-axis correction factors. The off-axis diffuse source correction is identical to the formula given in Equation (10) and Figure 7 of Tappin, Eyles, and Davies (2015). Also, it was not considered necessary to revise the large-scale flatfield corrections as these are not affected by the adjustments to the calibration parameters.

As for in the case of the HI-2 calibrations (Tappin, Eyles, and Davies, 2015), we determined the medians of all of the measured count rates for each star. We then computed a predicted count rate by folding the stellar spectra through the nominal (pre-launch) instrument parameters, and applied an estimated correction for the effect of orbital motion on the cosmic ray scrubbing; Appendix A.3, Equation (5). We then performed a weighted L1-norm linear fit of the measured rates to the predicted values, constrained to pass through the origin, to obtain the ratio of the measured rate to the predicted rate. Each input value was weighted by $N_{\text {obs }} / I Q$, where $N_{\text {obs }}$ is the number of measurements of a star. and $I Q$ is the interquartile range for the count rate measurements of that star. $N_{\text {obs }}$ was typically around 1400 for HI-1A and between 800 and 900 for HI-1B (this number is much lower than for HI-1A because of the rejection of high- $\Delta$ images). We estimated the $1 \sigma$ errors by the $\frac{1}{2} \pm \frac{1}{\sqrt{N_{\mathrm{s}}}}$ quantile fits where $N_{\mathrm{s}}$ is the number of stars used (Koenker and Hallock, 2001; Tappin, Eyles, and Davies, 2015).

The results of these fits are summarized in Table 2, along with the earlier determinations by Bewsher et al. (2010) and Bewsher, Brown, and Eyles (2012). 
Table 2. Fitted corrections to the pre-launch calibration parameters and previous determinations; the raw corrections (which are included for comparison) do not take account of the effect of orbital motion. BEA = Bewsher et al. (2010), BBE = Bewsher, Brown, and Eyles (2012).

\begin{tabular}{|c|c|c|c|c|c|c|}
\hline \multirow[t]{2}{*}{ Instrument } & \multirow{2}{*}{$\begin{array}{c}\text { Correction } \\
\qquad G_{0}\end{array}$} & \multirow[t]{2}{*}{ Error } & \multicolumn{2}{|c|}{ Raw } & \multirow[t]{2}{*}{ BEA } & \multirow[t]{2}{*}{$\mathrm{BBE}$} \\
\hline & & & Correction & Error & & \\
\hline HI- $1 \mathrm{~A}$ & 0.918 & 0.008 & 0.911 & 0.009 & 0.93 & 0.94 \\
\hline HI-1B & 0.990 & 0.008 & 0.982 & 0.008 & 0.98 & 1.00 \\
\hline
\end{tabular}

\subsection{The Temporal Variation of Gain}

Both Bewsher, Brown, and Eyles (2012) and Tappin, Eyles, and Davies (2015) grouped the data by STEREO orbit in their attempts to measure the degradation rates of the $\mathrm{HI}$ instruments. This grouping into orbits has two undesirable consequences:

i) Any data after the last completed solar orbit cannot be used.

ii) Each star is observed for only a few days once per orbit, so stars that pass through the field of view early in the orbit are measured at very different dates from those that pass though late in the orbit.

However, the method used by Tappin, Eyles, and Davies (2015) to reduce the spread in the orbital medians suggests the possibility of taking an alternative approach that removes the need to group the measurements, and thus eliminates these effects.

i) For each star, the median count rate for that star over the science phase of the mission up to the start of gyroless operations was determined.

ii) All the count rates for that star were normalized by this median value. A median date of the observations of each star was also computed, as was the median date of all the observations.

iii) A linear L1-norm fit to the normalized rates as a function of time was performed for each star.

iv) The normalization factor for each star was then adjusted using the computed slope to correct the median count rate from the star's median date to the median date of all of the measurements. The degradation rate is then the median of the values for all of the stars.

v) Steps iii) and iv) were repeated until the computed degradation rate converged to a stable value.

In Figure 2, we show 2D histograms of the normalized count rates resulting from applying the above procedure, with the fitted slopes overlaid. For HI-1A, the histogram suggests strongly that prior to about the start of 2009, there was little or no degradation. Therefore, for HI-1A, we performed the evolution analysis using only data from the start of 2009 onward, and show a constant extension of the 2009 value for earlier times. The error limits on the fits are small enough that they are comparable with the line widths in Figure 2. 
a)

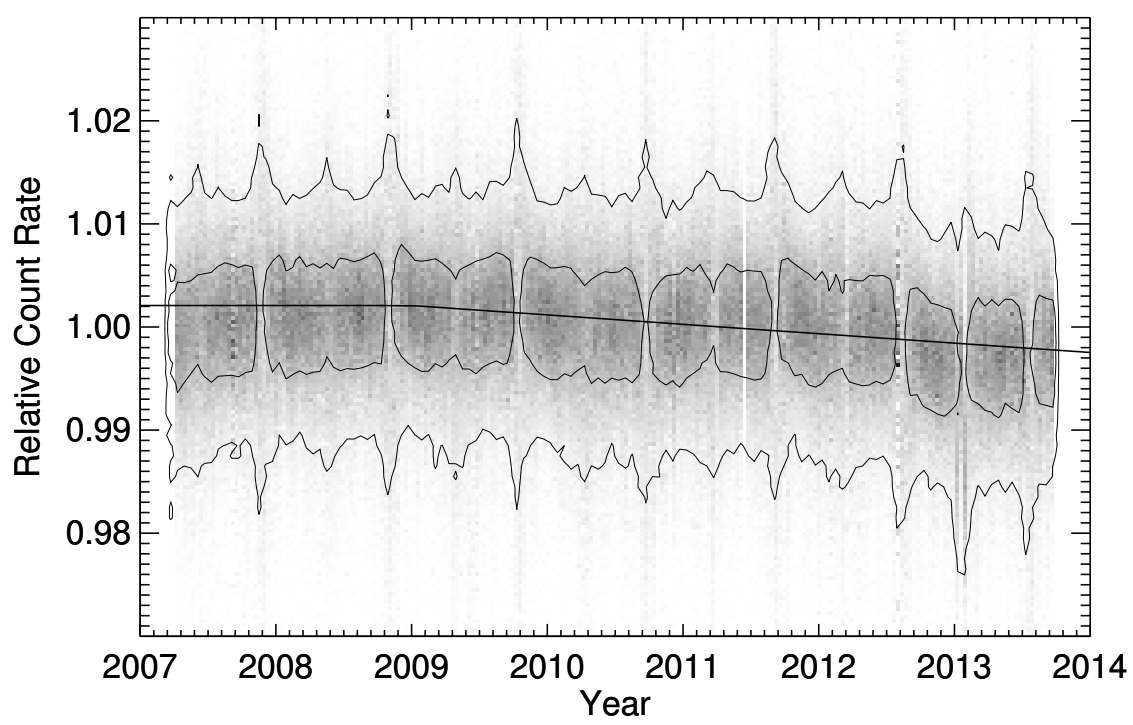

b)

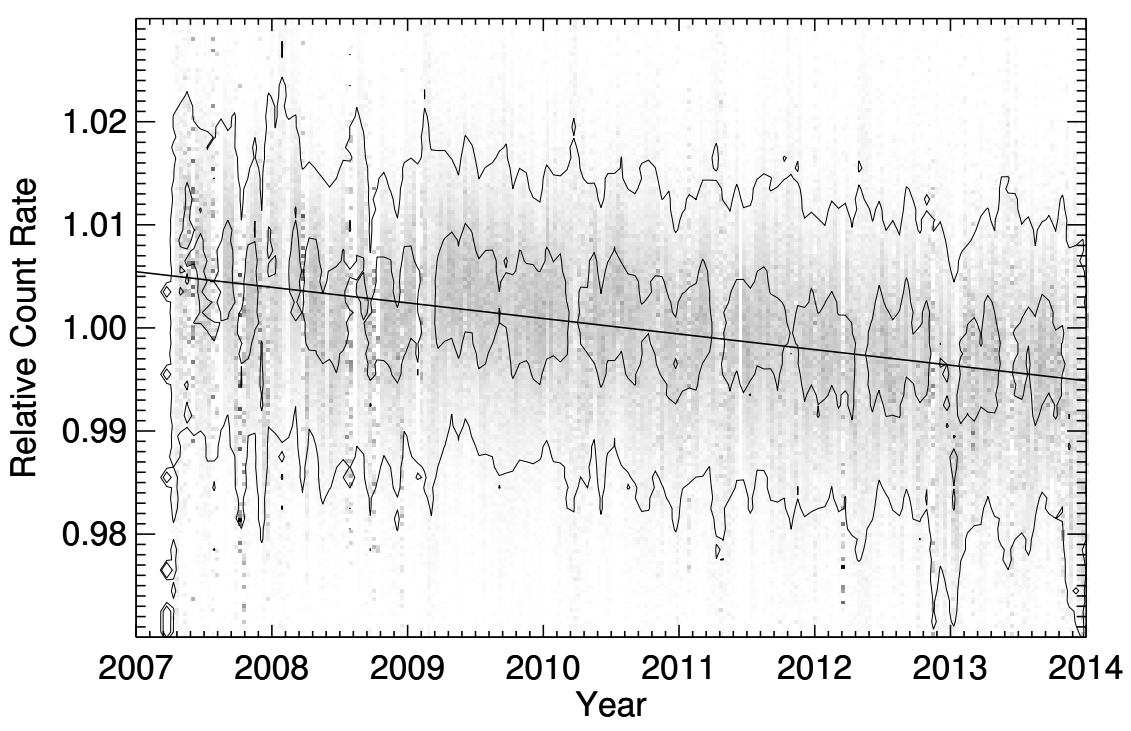

Figure 2. 2D histograms of evolution of stellar count rates as a function of time for the HI-1 instruments, using a photometry aperture of 3.0 bins. a) HI-1A, b) HI-1B. The histograms are shown as greyscale shading with overlayed contours. The linear fits from Equation (1) are overlaid. For HI-1A the value at 2009.0 is projected back as a constant value for dates prior to 2009.0. The approximately periodic broadenings apparent in the HI-1A distribution correspond to times when the field of view was at low galactic latitude. 
Table 3. HI-1 gain rates of change and adjustments to the origin for the HI-1 instruments, relative to the gains in Table 2.

\begin{tabular}{cccccc} 
Instrument & $\begin{array}{c}\text { Gain change } \\
\text { per year }\end{array}$ & Error & $\begin{array}{c}\text { Intercept at } \\
\text { calibration } \\
\text { origin } \\
{\left[F_{\mathrm{TC}}\right]}\end{array}$ & Error & $\begin{array}{c}\text { Gain } \\
\text { correction } \\
\text { at origin } \\
{\left[G_{\mathrm{TC}}\right]}\end{array}$ \\
\hline HI-1A & -0.000912 & 0.000013 & 1.00209 & 0.000036 & 0.920 \\
HI-1B & -0.001511 & 0.000003 & 1.00545 & 0.000013 & 1.044
\end{tabular}

To obtain an effective instrument gain at any date, we combine the results from Tables 2 and 3 as:

$$
G(T)=G_{\mathrm{TC}}\left(1+\frac{R}{F_{\mathrm{TC}}} \Delta T\right),
$$

where: $G_{\mathrm{TC}}=G_{0} F_{\mathrm{TC}}$ is the gain at the calibration origin (2009.0 for HI-1A and 2007.0 for HI-1B), $G_{0}$ is the gain value from Table $2, F_{\mathrm{TC}}$ is the origin intercept and $R$ is the degradation rate (both from Table 3 ), and $\Delta T$ is the time in years after the calibration origin. The gain correction to be applied to images to convert them to count rates at the origin, and thence to physical units, is the inverse of Equation (1):

$$
C(T)=\frac{1}{G(T)} \approx \frac{1}{G_{\mathrm{TC}}}\left(1-\frac{R}{F_{\mathrm{TC}}} \Delta T\right) .
$$

For both HI-1A and HI-1B, the approximation in Equation (2) is accurate to better than one part in $10^{4}$ for $\Delta T$ of ten years, which is significantly better than the accuracy of our measurements (and most probably the assumption of linear degradation).

In Table 4, we present the conversion factors from images in units of DN s ${ }^{-1}$ (CCD pixel) to various physical units. These are the same units that were presented by Tappin, Eyles, and Davies (2015) for HI-2; their derivation is described in detail there. The conversion factors in Table 4 are about four times larger than the corresponding values given by Bewsher et al. (2010) and Bewsher, Brown, and Eyles (2012) as those authors used units of $\mathrm{DN} \mathrm{s}^{-1}$ (image bin) ${ }^{-1}$; we prefer to use

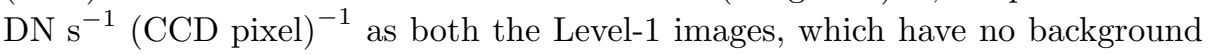
subtraction, and the background-subtracted Level-2 images that are available from UKSSDC are in those units. The factors presented in Table 4 are those applicable at the calibration origins, 2009.0 for HI-1A and 2007.0 for HI-1B. Following Tappin, Eyles, and Davies (2015), we present three versions of the SI unit conversions, for the whole spectrum using the solar constant of Kopp and Lean (2011), for the wavelength band covering the extreme limits of the HI sensitivity, and finally the actual energy detected by the HI-1 cameras. The latter two use the solar spectrum of Neckel and Labs (1984) to define the input spectrum. The $360-1080 \mathrm{~nm}$ value is the most comparable with the values quoted by Bewsher et al. (2010), but each has potential uses. The final row of 
Table 4. Conversion factors to obtain physical units from HI-1 images (in $\mathrm{DN} \mathrm{s}^{-1}$ (CCD pixel) $^{-1}$ ), at the calibration origin, and the rates of change thereof. SI units are $\mathrm{Wm}^{-2} \mathrm{sr}^{-1}$.

\begin{tabular}{lcc} 
Unit & HI-1A & HI-1B \\
\hline B $\odot$ & $3.63 \times 10^{-13}$ & $3.55 \times 10^{-13}$ \\
S10 & 806. & 790. \\
SI Units: $0-\infty$ & $7.25 \times 10^{-6}$ & $7.10 \times 10^{-6}$ \\
SI Units: $360-1080 \mathrm{~nm}$ & $5.17 \times 10^{-6}$ & $5.06 \times 10^{-6}$ \\
SI Units: Passband & $8.21 \times 10^{-7}$ & $7.55 \times 10^{-7}$ \\
Annual Change $\left(=-F_{\mathrm{TC}} / R\right)$ & 0.000910 & 0.001503
\end{tabular}

the table is the degradation rate to be applied to the origin factors (i.e. $-R / F_{\mathrm{TC}}$ from Equations (1) and (2)). To find the conversion factor at any date, the factor at the calibration time origin should be multiplied by $1-\Delta T R / F_{\mathrm{TC}}$. For HI- $1 \mathrm{~A}$ images prior to 2009.0, the value computed at 2009.0 is appropriate.

This evolution rate, along with the revised photometric calibration parameters, has been incorporated into the HI processing pipeline software.

\section{Discussion}

The gain correction that we determine for HI-1A is somewhat lower than the previous determinations, albeit only by about $1 \sigma$. Note that neither Bewsher et al. (2010) nor Bewsher, Brown, and Eyles (2012) quote estimates for the uncertainty of their parameters but they were of the order of $1 \%$. One possible source of the discrepancy is that Bewsher et al. (2010) imply that they used the fixed relationship between magnitude and flux given in Gray (2005). This slightly underestimates the flux of $\mathrm{K}$ and $\mathrm{M}$ class stars (which dominate the sample used) compared with integrating the spectrum over the V-passband as we have done. This will result in those authors obtaining a somewhat higher estimate for the effective gain of the instruments compared with the values presented in this article. The differences of date range between that used in our analysis and that used by Bewsher et al. (2010) and Bewsher, Brown, and Eyles (2012) also mean that we would expect our gains to be lower; however the degradation rates that we have measured are too low to account for more than a small fraction of the difference.

Our estimate of the HI-1B gain lies between those of Bewsher et al. (2010) and Bewsher, Brown, and Eyles (2012). However we might expect that we would obtain a higher gain in this case, as those authors were not aware of the stellarcount reduction caused by the particle-scrubbing algorithm at times when the HI-1B pointing was unstable (see Appendix A.2). We therefore consider that the effect of the particle scrubbing, and the corrections to the spectral integrations approximately cancel out for HI-1B.

The rates of degradation of the HI-1 cameras found here are comparable with those found for HI-2 (Tappin, Eyles, and Davies, 2015), and about an order of magnitude less than the upper limits determined by Bewsher, Brown, and Eyles 
(2012) and BenMoussa et al. (2013). Given the lack of degradation in HI-1A and the pointing instabilities in HI-1B prior to the start of 2009, we do not think that it would have been possible to obtain degradation rates from the data available to Bewsher, Brown, and Eyles (2012) (up to mid-2011). As in the case of HI-2, this slow degradation rate is very good news for the long-term operation of the $\mathrm{HI}$ instruments. While we do not have a definitive explanation for the excellent stability of all of the $\mathrm{HI}$ instrument responses, we think it very probable that the comparatively benign particle environment and low operating temperature (compared with the Solar Mass Ejection Imager, SMEI, in low Earth orbit) and developments in CCD technology between the construction of the Large Angle Spectroscopic COronagraph, LASCO, instruments and the HI instruments are the main reasons.

The degraded pointing stability of the spacecraft since the transitions of the spacecraft to gyroless operation presents a concern for calibration since the return of STEREO-A to full operations and after the hoped-for recovery of STEREO-B, as not only is the apparent gain from stellar photometry reduced, but the scatter in the measurements is increased (Appendix A.1). We must however emphasize that this has no impact on the measurements of heliospheric structures as these extend over many CCD pixels and so the pointing fluctuations do not produce changes that can trigger the particle scrubbing process. This is also true of the other effects discussed in Appendix A. The pointing degradation does, however, have implications for the future use of HI data for stellar studies. These are discussed in a separate article (Tappin, 2017). It is also clear that such effects as these need to be considered in the design and implementation of future heliospheric imagers and their host spacecraft.

The differences between the photometric calibration parameters found here, and the earlier determinations of Bewsher et al. (2010) and Bewsher, Brown, and Eyles (2012) are relatively small. We believe that these differences can be adequately explained because: i) for different reasons, both instruments show unusual behaviour prior to the start of 2009, and ii) the approximation used in determining stellar fluxes by Bewsher et al. (2010) and Bewsher, Brown, and Eyles (2012) is least accurate for the late-type stars that dominate the samples used.

\subsection{Note on the HI-2 Calibration}

When we carried out the calibration and degradation analysis for HI-2 (Tappin, Eyles, and Davies, 2015), we were not aware of this pointing degradation and of its effect on the stellar responses, as it only became apparent during the analysis for the work presented in this article. However we are confident that the results presented in Tappin, Eyles, and Davies (2015) are not significantly affected. For HI-2B, we did not use any data after the transition to gyroless operations, so there cannot be any effect. For HI-2A, we only used about four months of affected data for the overall gain determination, and about one month in the final orbit of the degradation determination. Since we were using medians and L1-norm fits and the degraded data represent only a small fraction of the total data included, the effects will therefore be minimal-smaller than the errors in 
the fit; we estimate about $0.1-0.2 \sigma$. The orbital motion effect is also negligibly small for HI-2. The pointing jumps, and associated apparent gain dips, seen in HI-1B do not occur in HI-2B.

\section{Summary}

In conclusion we may summarize our results as:

i) We have found that there has been a very small but measurable degradation of the HI- 1 sensitivities through the STEREO mission up to superior conjunction. This degradation, of about $0.08 \%$ per year for HI- $1 \mathrm{~A}$ and $0.15 \%$ per year for HI-1B, is comparable with the values obtained for the HI-2 instruments (Tappin, Eyles, and Davies, 2015). It is about an order of magnitude slower than the upper limits found by Bewsher, Brown, and Eyles (2012) and BenMoussa et al. (2013), and also substantially lower than the rates found for other white light imaging instruments, LASCO C2 (Llebaria, Lamy and Danjard, 2006), LASCO C3 (Thernisien et al., 2006), and SMEI (Buffington et al., 2007).

ii) We find that small $\approx 1 \%$ adjustments should be made to the previouslypublished photometric calibration parameters for the HI-1 instruments. These have been implemented in the processing pipeline software, along with the degradation rates.

iii) We have also found that stellar count-rate measurements from the HIs can be affected by the cosmic ray scrubbing procedures when the pointing stability is reduced, even when the pointing control is within the spacecraft requirements. We also find that the motion of the stars across the field of view as the spacecraft orbits the Sun has a small effect. This does not affect measurements of the corona or of solar wind disturbances in any way, but it does have implications for future calibrations and for stellar studies. We emphasize that the fact that these effects are detectable and have had to be taken into consideration is a testament to the outstanding performance of the HI cameras, rather than a deficiency.

Acknowledgments The Heliospheric Imager (HI) instruments were developed by a collaboration that included the Rutherford Appleton Laboratory and the University of Birmingham, both in the United Kingdom, and the Centre Spatial de Liège (CSL), Belgium, and the US Naval Research Laboratory (NRL),Washington DC, USA. The STEREO/SECCHI project is an international consortium of the Naval Research Laboratory (USA), Lockheed Martin Solar and Astrophysics Lab (USA), NASA Goddard Space Flight Center (USA), Rutherford Appleton Laboratory (UK), University of Birmingham (UK), Max-Planck-Institut für Sonnensystemforschung (Germany), Centre Spatial de Liège (Belgium), Institut d'Optique Théorique et Appliquée (France), and Institut d'Astrophysique Spatiale (France).

We thank G.J. White for valuable discussions about aperture photometry.

Support for this work was provided by the UKSA STEREO Post Launch support.

Disclosure of Potential Conflicts of Interest The authors declare that they have no conflicts of interest. 


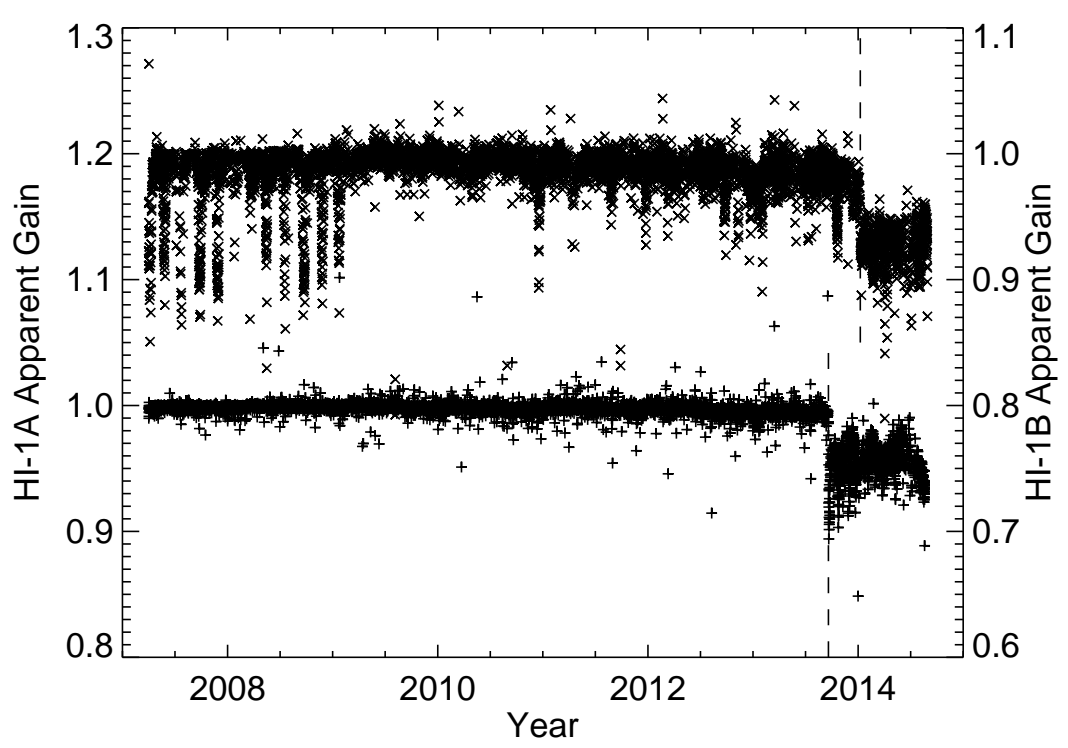

Figure 3. The apparent gains of the HI-1 cameras over the course of the mission up to the start of conjunction operations. Each point represents the median value for a single star as it transited the HI-1 field during one orbit. The + symbols and the left axis show the values for HI-1A, the $\times$ symbols and the right axis those for HI-1B. The vertical dashed lines show the times of the transitions to gyroless operations.

\section{Appendix}

\section{A. Gain Anomalies}

During the early development of the techniques used in this article to determine the degradation rates of the HI-1 cameras, disagreements between some of the methods that we tested suggested that there must be some peculiarities in the evolution of the instrument gains. In this Appendix we present a brief summary of the anomalies and their implications for the analyses presented in this article as well as for the use of the HI data for scientific analysis.

In Figure 3 we plot the per-orbit median count rates of each star scaled by the whole-mission median for that star. Two features are immediately apparent:

i) There was an abrupt drop in the apparent gain of both HI-1 instruments late in the mission. A similar drop (not shown) was also seen in the apparent gain of the HI-2 instruments.

ii) There were a number of drops in the apparent gain of HI-1B, predominantly before the start of 2009. These drops appear to be quasi-periodic.

Analysis of the F-coronal signal in the Level-1 images (which have no background subtraction) shows that these gain anomalies do not affect the response to extended sources, only that to point sources. 


\section{A.1. Spacecraft Attitude Control}

The late-mission drops in apparent gain occurred (to the nearest day) at the times at which the attitude-control gyros were switched off (18 September 2013 for STEREO-A, and 7 January 2014 for STEREO-B). After this time, attitude sensing was maintained with the guide telescope and star tracker. During gyroless operation, the pointing stability of the spacecraft (especially in roll) is significantly degraded, from a standard deviation on a timescale of one hour of less than 1 arcsecond to about 1 arcminute with maximum excursions of about $0.1^{\circ}$. It should however be noted here that this remains well within the mission specification of a roll jitter of better than 3.4 arcminutes on a timescale of 15 to 30 seconds (Driesman, Hynes and Cancro, 2008).

Prior to transmission to Earth, the HI- 1 science images are $2 \times 2$ binned and then 30 (99 for HI-2) separate exposures are summed (Eyles et al., 2009). A cosmic-ray scrubbing algorithm is applied to each exposure before binning and summing to remove energetic-particle hits (Eyles et al., 2009). This algorithm flags any pixel in an exposure that is more than $5 \sigma$ above its value in the previous exposure and replaces it with the value from the previous exposure, i.e.,

$$
I_{c}^{\prime}= \begin{cases}I_{\mathrm{p}} ; & \text { Where } \\ I_{\mathrm{c}} ; & \text { Elsewhere }\end{cases}
$$

where $I_{\mathrm{c}}$ is the number of counts in a pixel in the current exposure, $I_{\mathrm{p}}$ is the number of counts in the same pixel in the previous exposure and $I_{\mathrm{c}}^{\prime}$ is the number of counts in the pixel in the corrected exposure. The factor of $\sqrt{15}$ comes from the fact that $1 \mathrm{DN}$ corresponds to 15 photoelectrons (Eyles et al., 2009). The scrubbing is only triggered by increases in counts relative to the previous image because cosmic-ray hits always add counts to the image, therefore $I_{\mathrm{c}}^{\prime} \leq I_{\mathrm{c}}$. Examination of the particle-hit statistics showed that after the change to gyroless attitude control, the number of flagged pixels increased substantially. We therefore conclude that the degraded pointing stability, which can lead to shifts of up to about one image bin near the centre of the image for both HI1 and HI-2 over the timescale of the image accumulation, is causing the steep flanks of stellar signals in the HI images to be eroded by the particle scrubbing algorithm.

On 24 February 2016 a new attitude-control algorithm was implemented on STEREO-A, which gives significantly improved pointing stability. Initial tests show that this will probably be sufficient to allow meaningful gain determinations for the post-conjunction phase of the mission, but it will certainly require a longer interval to obtain meaningful numbers than was the case while the gyros were in use. As of the time of writing there is not a sufficient interval of observations to make a useful determination.

\section{A.2. HI-1B Movements}

The cause of the short-duration drops in the apparent gain in HI-1B is less immediately obvious. However it was noted that the majority of them occurred 
close to the times of spacecraft momentum dumps (W.T. Thompson, private communication, 2015). A closer analysis shows that each gain drop generally starts a few days prior to the dump and continues until a few days after. The gain drop does not correlate well with any measure of the spacecraft pointing or fluctuations thereof. It has also been found (Brown, Bewsher, and Eyles, 2009; Davis et al., 2012) that the HI-1B pointing undergoes sudden shifts of several image bins, which were believed to be caused by dust impacts; in fact the distribution of such shifts was used by Davis et al. (2012) to estimate the dust distribution near $1 \mathrm{AU}$.

In Figure 4 we show the pointing offsets at the centre of the HI-1B field for the science phase of the mission from 1 April 2007 until the loss of contact with STEREO-B on 1 October 2014. These offsets are calculated by comparing the nominal pointing information in the Level-0 image headers with the corrected pointings in the Level-1 image headers (Brown, Bewsher, and Eyles, 2009). This shows that rather than being a simple movement between two extremes, there is a D-shaped locus of accessible pointings and that the pointing is usually close to the periphery of this. The offset double-D shape is the result of a correction to the STEREO-B roll calibration that was applied on 18 June 2008 (W.T. Thompson, private communication, 2015). This correction resulted in a change of the nominal pointing placed in the Level-0 image headers. A corresponding change in the pointing adjustments at this time is also present in the HI-2B data. The other movements seen in Figure 4 occur in HI-1B alone and so must be related to movements of the HI-1B camera mounting, relative to the rest of the HI-B structure.

While the times of low apparent gain in HI-1B, like the gyroless data for all imagers, correspond to times of high particle hit detections, there is also a slow variation of the hit rates around the orbit due to the variation in the number of stars as a function of galactic latitude (see Appendix A.3). Therefore, setting a simple threshold for particle hits per image would either fail to flag some affected images or would exclude some images unnecessarily. Therefore to find a proxy for times when the particle scrubbing was causing significant erosion of stellar counts, but handling the slower variations in a meaningful way, we devised the statistic $\Delta$. We define $\Delta$ as:

$$
\Delta(P)=\frac{P_{0.75}}{\operatorname{mmm}\left(P_{0.25}, 180\right)}-1,
$$

where $P$ represents the number of pixels scrubbed in each exposure of an image, $P_{x}$ represents the $x$ th fractile of those counts and $\operatorname{mmm}\left(P_{x}, n\right)$ represents the background of $P_{x}$ computed using the $\mathrm{mmm}$ routine from the SolarSoft DAOPHOT suite from the $n$ images before and after the current image; the \pm 180 image window corresponds to \pm five days at the nominal HI- 1 image cadence of 40 minutes, and was chosen to give a baseline shorter than the slow variations of recorded particle hits around the orbit, but longer than the typical gain dropouts. This parameter was found to correlate well with times of reduced apparent gain and to be largely insensitive to slow variations of hit rates around the orbit. The value of $\Delta$ also increased very markedly for all of the HI cameras following the switch to gyroless attitude control (although those 


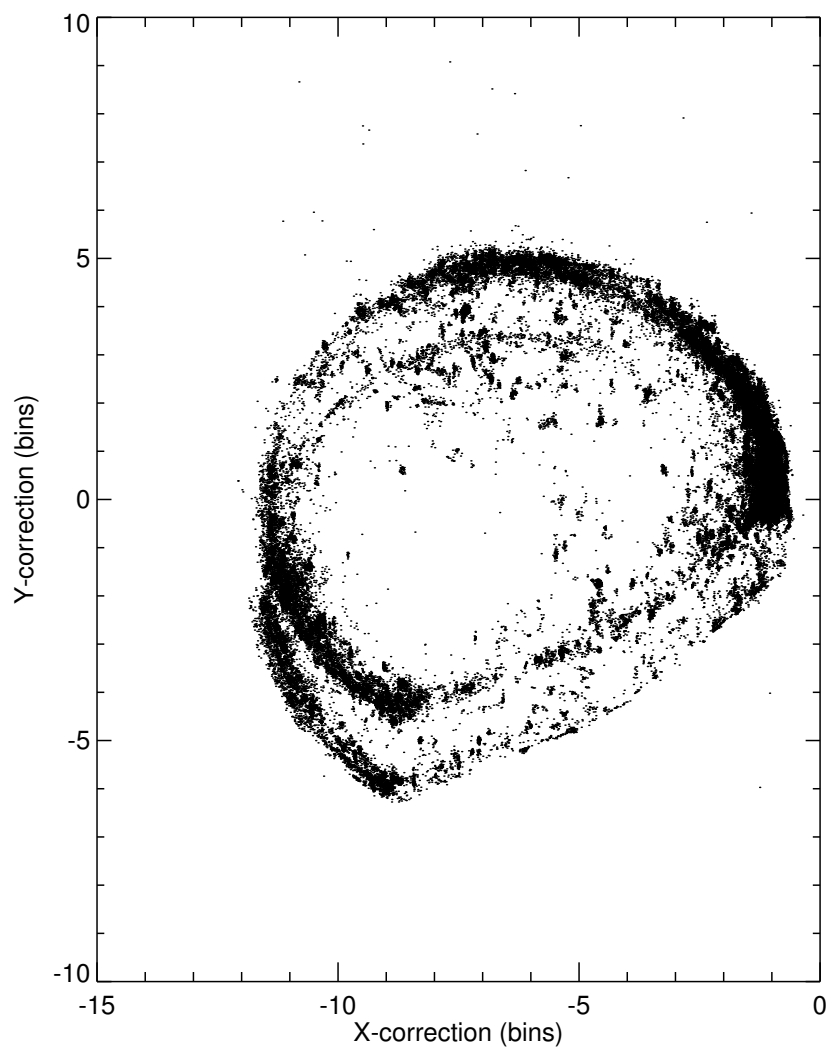

Figure 4. Pointing offsets of HI-1B relative to its nominal pointing from 1 April 2007 to 1 October 2014. Each dot represents the pointing offset of a single HI-1B science image. All images within the interval are included. The points well outside the main locus are from images where the pointing determination (Brown, Bewsher, and Eyles, 2009) did not find a satisfactory solution - such images are not processed through to the Level-2 stage and thus are not included in the main analysis presented in this article.

data are excluded from the analysis in the main body of this article). After some experimentation, we chose to exclude any images where $\Delta$ exceeded 0.15 from use in the analyses presented in this article. This eliminated about 39,000 of 86,500 science images for HI-1B, and about 6000 of 83,000 for HI-1A (the latter are most probably real particle events with short enough timescales to produce a high value of $\Delta$ ).

To better understand the behaviour of HI-1B across a momentum dump, we present plots of the apparent gain, and of several parameters related to the particle hit counts and the pointing of HI-1B in Figure 5. In Figure 5a we show the evolution of the apparent gain of HI-1B across the momentum dump; the reduced gain both before and after the actual time of the dump is clearly seen. In Figure $5 \mathrm{~b}$ we plot the values of $\Delta$ over the same interval, showing how low gain matches up with times of high particle hit spread. Figure $5 \mathrm{c}$ shows the 


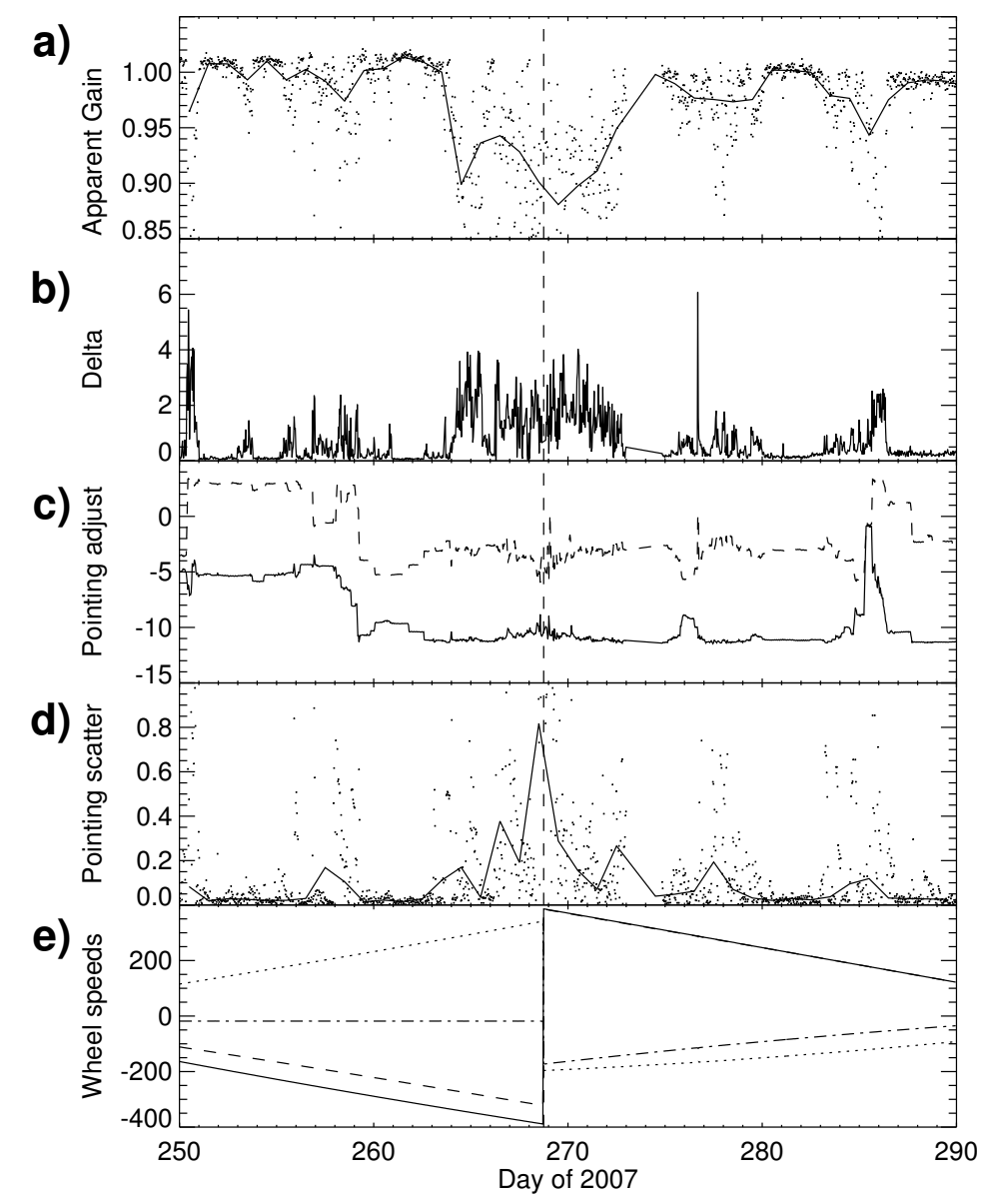

Figure 5. Example of the depression of HI-1B apparent gain around the momentum dump on 25 September 2007 (day 268). a) The apparent gain of HI-1B. The continuous line is the daily average, the dots are values for individual images and the vertical dashed line marks the time of the momentum dump. b) The cosmic-ray spread parameter $[\Delta]$. c) The adjustments of pointing made between the Level-0 and Level-1 images in image bins (Brown, Bewsher, and Eyles, 2009). The continuous line is the correction in the image $x$-direction and the dashed line is in the $y$-direction. d) The pointing scatter (defined as the pointing correction for the current image minus a 15-image running median of the correction). The line is a daily median value and the points are the values for each image, also in image bins. e) The speeds of the four STEREO-B reaction wheels (obtained from the STEREO Science Center: stereo-ssc.nascom.nasa.gov/data/moc_sds/behind/data_products/converted_sc_ housekeeping/), in rps.

pointing corrections between the Level-0 and Level-1 images. The large shifts discussed by Brown, Bewsher, and Eyles (2009), correspond to rapid movements across the D-shaped region of accessible pointings, and they appear as the major discontinuities in the pointing corrections in Figure 5c. In addition to these large jumps there are intervals of smaller fluctuations on timescales less than or comparable with the HI-1 image cadence (Figure $5 \mathrm{c}$ and $\mathrm{d}$ ). We found that there 


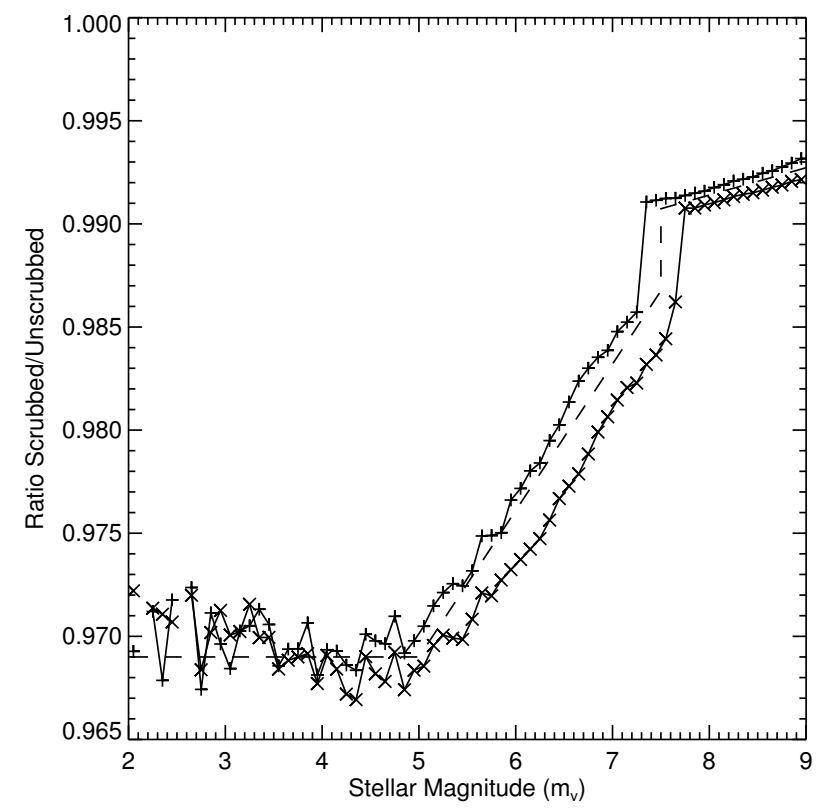

Figure 6. Estimated effect of the cosmic ray scrubbing on stellar count rates in HI-1 images due to the normal movement of the stars across the CCD. The line with $\times$ symbols shows the results for HI-1A, the line with + symbols shows HI-1B. The dashed line shows the estimated correction given in Equation (5).

was good correlation between these rapid fluctuations in the pointing correction and times of low apparent gain (and high $\Delta$ ). These times of rapid pointing fluctuation occur preferentially whenever any of the attitude-control reaction wheels were spinning faster than 350 revolutions per second (rps) (Figure 5e), but are they also seen at other times, still mainly when the wheel speed was high. The reduction in the occurrence of these gain drops after the start of 2009 corresponds to an increase in the frequency of momentum dumps so that after this time the wheels were seldom spun up to $350 \mathrm{rps}$. We speculate that vibrations from the reaction wheels cause whatever is loose in HI-1B to break free at spin rates above $350 \mathrm{rps}$. At somewhat lower wheel speeds the same vibrations, while insufficient to initiate wandering, do prevent the assembly from settling after it has been freed by some other cause such as a dust impact.

\section{A.3. Orbital Motion}

A final consideration is the extent to which the normal drift of the stars across the image plane will trigger false positives in the cosmic-ray scrubbing procedure. Since there is no record retained of exactly which pixels have been scrubbed, only the total number in each exposure, this can only be estimated by simulation. On most days, one single-exposure $2048 \times 2048$ image is transmitted for calibration and instrument monitoring purposes. These calibration images can potentially be used as the basis for a simulation of the interaction of the apparent stellar motion 
with the scrubbing algorithm. This was done by generating simulated shifted images from the actual single-exposure images, and then applying the scrubbing algorithm to these image pairs. For this investigation, we took a number of these full-resolution single exposures (specifically those from July and August of 2009, 43 for HI-1A and 42 for HI-1B) and simulated the cosmic ray scrubbing, and hence its effect on stellar count rates, by using the algorithm below:

i) For each single-exposure image, the previous exposure was simulated by shifting 2.5 or 25.0 arcseconds along the $x$-axis (corresponding to the one minute exposure cadence during an image accumulation, and the ten minute gap between the last exposure of one image and the first exposure of the next image, respectively), using the IDL routine INTERPOL to perform a spline interpolation.

ii) Equation (3) was then used to compute the scrubbed current exposure, with the simulated image serving as the previous exposure.

iii) Both the original and scrubbed current images were binned to a resolution of $1024 \times 1024$

iv) For all stars brighter than magnitude 9.0 (including those not suitable for normal calibration purposes), the aperture-photometry methods described in Section 2.2 were used to compute the counts.

v) The ratios of the scrubbed to the unscrubbed integrated stellar counts were then computed.

vi) Finally an estimated effect for a normal science image was derived by summing the 25 and 2.5 arcsec scrubbing ratios with weights of $1 / 30$ and 29/30 respectively.

In Figure 6, we show the simulated ratios of scrubbed to unscrubbed count rates, binned in 0.1 magnitude bins. The simulations show a small discrepancy between HI-1A and HI-1B, for which we do not currently have an explanation. Since this is a small adjustment to a small correction we feel justified in using a single intermediate value to make an estimate of the fractional reduction of count rate due to the effects of orbital motion. A reasonable approximation can be made with three linear relations:

$$
\frac{R_{\text {scrubbed }}}{R_{\text {unscrubbed }}}=\left\{\begin{array}{l}
0.981+0.0013 m_{v} \\
0.933+0.0071 m_{v} \\
0.989
\end{array}\right\} \text { where }\left\{\begin{array}{l}
7.5 \leq m_{v}<9.0 \\
5.0 \leq m_{v}<7.5 \\
m_{v}<5.0
\end{array},\right.
$$

this relation is shown as the dashed line in Figure 6. Although this effect appears substantial, the majority of the stars used in the determination of the calibration parameters are towards the faint end of the range (only about 500 of almost 1500 stars used in the calibrations are brighter than magnitude 7.5, and fewer than 50 are brighter than 6.0); therefore the influence is dominated by the first condition in Equation (5), and the resulting adjustment to the calibration parameters is slightly less than $1 \%$. Since the scrubbing due to orbital motion is a function only of stellar magnitude, it affects only the photometric calibration parameters and not evolution rates.

We also note that the calibration parameters for HI-2 presented by Tappin, Eyles, and Davies (2015) are not materially affected by this effect as: i) the 
movement of the stars across the CCD is about four times slower (in pixels per exposure) because of HI-2's larger pixel size, ii) only one in 99 exposures has a long gap before it rather than one in 30, and iii) the PSFs for HI-2 (especially HI-2B) are larger than in HI-1 so the image gradients are smaller. As a result of these any corrections in HI-2A, even for the brightest stars, are only about $0.2 \%$ (and much less than that for the majority of stars), while in HI-2B there is no detectable effect. We therefore conclude that there is no need for a revision to the calibration parameters presented by Tappin, Eyles, and Davies (2015).

\section{A.4. Summary}

The effects outlined in this Appendix are described in more detail in a separate article (Tappin, 2017) as they are of considerable importance for the use of HI data for astronomical studies.

We emphasize here that the only influence that these effects have on the primary scientific objectives of the STEREO-HI cameras (the study of structures in the solar wind) is in the determination of the calibration parameters used to convert the observations into physical units. We have taken them into account, by

i) excluding data after the switch to gyroless pointing control,

ii) excluding times when high values of $\Delta$ indicated HI-1B pointing shifts and

iii) applying a simple correction to compensate for the influence of orbital motion.

\section{References}

BenMoussa, A., Gissot, S., Schühle, U., Del Zanna, G., Auchère, F., Mekaoui, S., Jones, A.R., Walton, D., Eyles, C.J., Thuillier, G., Seaton, D., Dammasch, I.E., Cessateur, G., Meftah, M., Andretta, V., Berghmans, D., Bewsher, D., Bolsée, D., Bradley, L., Brown, D.S., Chamberlin, P.C., Dewitte, S., Didkovsky, L.V., Dominique, M., Eparvier, F.G., Foujols, T., Gillotay, D., Giordanengo, B., Halain, J.P., Hock, R.A., Irbah, A., Jeppesen, C., Judge, D.L., Kretzschmar, M., McMullin, D.R., Nicula, B., Schmutz, W., Ucker, G., Wieman, S., Woodraska, D., Woods, T.N.: 2013, Solar Phys. 288, 389. ADS. DOI.

Bewsher, D., Brown, D.S., Eyles, C.J.: 2012, Solar Phys. 276, 491. ADS. DOI.

Bewsher, D., Brown D.S., Eyles, C.J., Kellet, B.J., White, G.J., Swinyard, B.: 2010, Solar Phys. 264, 433. ADS. DOI.

Brown, D.S., Bewsher, D., Eyles, C.J.: 2009, Solar Phys. 254, 185. ADS. DOI.

Buffington, A., Morrill, J.S., Hick, P.P., Howard, R.A., Jackson, B.V., Webb, D.F.: 2007, (SPIE) CS-6689, 66890B. ADS. DOI.

Davis, C.J., Davies, J.A., St Cyr, O.C., Campbell-Brown, M., Skelt, A., Kaiser, M., MeyerVernet, N., Crothers, S., Lintott, C., Smith, A., Bamford, S., and Baeten, E.M.L.: 2012, Mon. Not. Roy. Astron. Soc. 420, 1355. ADS DOI

Driesman, A., Hynes, S., Cancro, G.: 2009, Space Sci. Rev. 136, 17. ADS. DOI.

Eyles, C.J., Harrison, R.A., Davis, C.J., Waltham, N.R., Shaughnessy, B.M., Mapson-Menard, H.C.A., Bewsher, D., Crothers, S.R., Davies, J.A., Simnett, G.M., Howard, R.A., Moses, J.D., Newmark, J.S., Socker, D.G., Halain, J.-P., Defise, J.-M., Mazy, E., Rochus, P.: 2009, Solar Phys. 254, 387. ADS. DOI.

Gray, D.F.: 2005, The observation and analysis of stellar photospheres Third edition, Cambridge U.P. Cambridge, (p211).

Hoffleit, D. and Warren, W.H., Jr.: 1995, VizieR Online Data Catalog 5050, vizier.cfa.harvard. edu/viz-bin/VizieR?-source=V/50 ADS. 
Howard, R.A., Moses, J.D., Vourlidas, A., Newmark, J.S., Socker, D.G., Plunkett, S.P., Korendyke, C.M., Cook, J.W., Hurley, A., Davila, J.M., Thompson, W.T., St Cyr, O.C., Mentzell, E., Mehalick, K., Lemen, J.R., Wuelser, J.P., Duncan, D.W., Tarbell, T.D., Wolfson, C.J., Moore, A., Harrison, R.A., Waltham, N.R., Lang, J., Davis, C.J., Eyles, C.J., Mapson-Menard, H., Simnett, G.M., Halain, J.P., Defise, J.M., Mazy, E., Rochus, P., Mercier, R., Ravet, M.F., Delmotte, F., Auchere, F., Delaboudiniere, J.P., Bothmer, V., Deutsch, W., Wang, D., Rich, N., Cooper, S., Stephens, V., Maahs, G., Baugh, R., McMullin, D., Carter, T.: 2008, Space Sci. Rev. 136, 67. ADS. DOI.

Kaiser, M.L., Kucera, T.A., Davila, J.M., St. Cyr, O.C., Guhathakurta, M., Christian, E.: 2008, Space Sci. Rev. 136, 5. ADS. DOI.

Koenker, R., Hallock, K.F.: 2001, J. Econ. Perspect. 15, 143.

Kopp, G., Lean, J.L.: 2011, Geophys. Res. Lett. 38, L01706. ADS. DOI.

Llebaria, A., Lamy, P., Danjard, J.-F.: 2006, Icarus 182, 281. ADS. DOI.

Myers, J.R., Sande, C.B., Miller, A.C., Warren, W.H., Jr., Tracewell, D.A.: 2001, VizieR Online Data Catalog 5109, vizier.cfa.harvard.edu/viz-bin/VizieR?-source=V/109. ADS.

Neckel, H., Labs, D.: 1984, Solar Phys. 90, 205. ADS. DOI.

Pickles, A.J.: 1998, Pub. Astron. Soc. Pac. 110, 863. ADS. DOI.

Scheuer, P.A.G.: 1957, Proc. Camb. Phil. Soc. 53, 764. ADS. DOI.

Stetson, P.B.: 1987, Pub. Astron. Soc. Pac. 99, 191. ADS. DOI.

Tappin, S.J.: 2017 Astron. J. Submitted.

Tappin, S.J., Eyles, C.J., Davies, J.A.: 2015, Solar Phys. 290, 2143. ADS, DOI.

Thernisien, A.F., Morrill, J.S., Howard, R.A., Wang, D.: 2006, Solar Phys. 233, 155. ADS. DOI.

Zacharias, N., Monet, D.G., Levine, S.E., Urban, S.E., Gaume, R., Wycoff, G.L.: 2004, Bull. Am. Astron. Soc., 36, 1418. ADS. 